\title{
Xaf1 can cooperate with TNF $\alpha$ in the induction of apoptosis, independently of interaction with XIAP
}

\author{
Yan Xia, ${ }^{1}$ Rachel Novak, ${ }^{1}$ Jennifer Lewis, ${ }^{2}$ Colin S. Duckett ${ }^{2}$ and \\ Andrew C. Phillips ${ }^{1}$ \\ ${ }^{1}$ Medical College of Georgia, Institute of Molecular Medicine and Genetics, CB2803, 1120 15th Street, Augusta, GA 30912, \\ USA; ${ }^{2}$ University of Michigan, Departments of Pathology and Internal Medicine, University of Michigan Medical School, \\ Room 5315, 1301 Catherine, Ann Arbor, MI 48109-0602, USA
}

Received 14 October 2005; accepted 28 November 2005

\begin{abstract}
XIAP-associated factor 1 (Xaf1) binds XIAP and re-localizes it to the nucleus, thus inhibiting XIAP activity and enhancing apoptosis [1]. Xaf1 expression is reduced or absent in tumor samples and cell lines suggesting it may function as a tumor suppressor [2-5]. To further study Xaf1 function we generated Xaf1 inducible cells in the osteosarcoma cell line Saos-2. Despite Xaf1 inducing apoptosis that is dramatically enhanced by TNF $\alpha$ we find no evidence for an interaction between Xaf1 and XIAP. Furthermore, Xaf1 expression sensitized XIAP ${ }^{-/-}$fibroblasts to TNF $\alpha$, demonstrating the existence of a novel mechanism of Xaf1 induced apoptosis distinct from antagonizing XIAP. Xaf1 expression promotes cytochrome c release that cannot be blocked by inhibition of caspase activity. This implicates a role for the mitochondrial apoptotic pathway, consistent with the ability of Bcl 2 to block Xaf1 induced apoptosis. The data indicate that in Saos2 cells Xaf1 activates the mitochondrial apoptotic pathway to facilitate cytochrome $\mathrm{c}$ release, thus amplifying apoptotic signals from death receptors. (Mol Cell Biochem 286: 67-76, 2006)
\end{abstract}

Key words: Xaf1, XIAP, TNF $\alpha$, cytochrome c, caspase, Bcl2, tumor suppressor

\section{Introduction}

Apoptosis is an evolutionarily conserved form of cell death, which plays critical roles during development, differentiation and tissue homeostasis of multi-cellular organisms. Defects in apoptosis play an important role in the pathogenesis of a number of diseases including cancer, neurodegenerative and auto-immune diseases [6]. Central mediators of the apoptotic process are the caspases. These proteases are synthesized as zymogens that are activated by either an increase in local concentration or by cleavage by other caspases (or in some cases non-caspase proteases) [7]. This can lead to a cascade of caspase activation, eventually mediating the orderly destruction of the cell [7, 8].

Two major pathways of apoptosis are described as the death receptor (or extrinsic) and the mitochondrial (or intrinsic). In both pathways apical initiator caspases are activated which in turn activate other caspases. The extrinsic pathway is activated by ligation of death receptors such as Fas and Tumor Necrosis Factor Receptor (TNFR) with their appropriate ligand. This results in the formation of a signaling complex activating caspase 8 [9]. The intrinsic or mitochondrial pathway is regulated by members of the $\mathrm{Bcl} 2$ family, which either promote or inhibit apoptosis $[10,11]$. This pathway 
can be activated by a variety of stresses including radiation and chemotherapeutic agents resulting in the release of cytochrome $\mathrm{c}$ and other pro-apoptotic molecules from the mitochondria into the cytosol. Cytochrome c binds to Apaf1 forming a macromolecular structure termed the apoptosome that recruits and activates caspase 9 [12].

Apoptosis is regulated post activation of caspases, by the inhibitor of apoptosis proteins (IAPs), that bind and inhibit the activity of caspases [13]. In many experimental systems, the X-linked inhibitor of apoptosis (XIAP) is the most potent of the IAPs, binding and inhibiting the activity of several caspases, notably caspases 3,7 and 9 [14, 15]. In addition to the activation of caspases, efficient induction of apoptosis requires relief of IAP-mediated caspase inhibition. A number of proteins that bind and antagonize IAP function have been identified including the mitochondrial sequestered SMAC/Diablo and HtrA2/Omi [16, 17]. These proteins are released from the mitochondria in response to apoptotic signals and inhibit IAP function.

In addition to these mitochondrial sequestered proteins, other proteins have been identified that can modulate XIAP activity, including Xaf1. Xaf1 was isolated as an XIAP interacting protein in a yeast 2-hybrid screen and antagonizes XIAP caspase inhibition by direct interaction and relocalization of XIAP from cytosol to the nucleus [1, 18]. Xaf1 expression has been found to be reduced or absent in multiple tumor cell lines, in comparison to normal cells [4]. In addition, expression is lost in a subset of gastric, melanoma and colon cancer samples, suggesting that Xaf1 may function as a tumor suppressor $[2,3,5]$.

To further investigate Xaf1 function we generated Xaf1 inducible Saos-2 cell lines. Doxycycline treatment results in accumulation of Xaf1 in the nucleus and apoptosis that is enhanced by treatment with other inducers of apoptosis, most dramatically TNF $\alpha$. Despite Xaf1 promoting apoptosis in this cell line, XIAP remains in the cytosol, and we see no evidence of an interaction between these proteins. In addition, expression of Xaf1 in XIAP null fibroblasts renders these cells sensitive to $\mathrm{TNF} \alpha$ treatment, demonstrating a XIAP-independent function of Xaf1. Furthermore we demonstrate that Xaf1 can induce apoptosis by activation of the mitochondrial pathway, promoting cytochrome $\mathrm{c}$ release that cannot be inhibited by the caspase inhibitor z-VAD. Our data reveal the existence of a previously unidentified function of Xaf1 in facilitating cytochrome c release to enhance apoptosis by other signals.

\section{Materials and methods}

\section{Transfection and cell culture}

Saos-2 cells and mouse fibroblasts were maintained in Dulbecco's modified Eagle's medium supplemented with
$10 \%$ fetal bovine serum, $2 \mathrm{mM}$ L-glutamine (Cellgro), $100 \mathrm{U} / \mathrm{ml}$ penicillin and streptomycin (Cellgro). For transfections, $5 \times 10^{5}$ cells were transfected with $10 \mu \mathrm{g}$ of expression plasmid and $2 \mu \mathrm{g}$ of pCMV CD20 expression plasmid by calcium phosphate precipitation. Xaf1 inducibles were generated using methods previously described [19, 20]. Parallel transfections allowed isolation of Xaf1 inducibles and cells containing only the empty vector as controls. The cells were maintained in Dulbecco's Modified Eagle's Medium supplemented with $10 \%$ fetal bovine serum (Tet System Approved FBS, BD Biosciences), 2 mM L-glutamine (Cellgro), $100 \mathrm{U} / \mathrm{ml}$ penicillin and streptomycin (Cellgro).

\section{Immunoprecipitations and immunoblotting}

Cells were harvested in Cell Lysis Buffer (20 mM Tris pH 7.4, $150 \mathrm{mM} \mathrm{NaCl}, 1 \mathrm{mM}$ EDTA, $1 \mathrm{mM}$ EGTA, $1 \%$ Triton $\mathrm{X} 100$ and complete proteinase inhibitors). Cellular debris was removed by centrifugation at $13000 \mathrm{~g}$ at $4^{\circ} \mathrm{C}$ for 10 minutes. For immunoprecipitations, the cell lysates were pre-cleared with $20 \mu \mathrm{l}$ of protein A-sepharose beads in Cell Lysis Buffer (Pierce, 20398), and then incubated with appropriate antibodies at $4^{\circ} \mathrm{C}$ for 2 hours, followed by addition of $20 \mu \mathrm{l}$ protein A-Sepharose beads and incubation at $4^{\circ} \mathrm{C}$ for 30 minutes. Immunoprecipitates were washed 4 times with Cell Lysis Buffer and once with PBS. Bound proteins were eluted and resolved by SDS-PAGE, and transferred to PVDF membranes (Hybond-P, Amersham). Membranes were blocked in TBST containing 5\% skimmed milk, and then incubated for 2 hours with specific antibodies (anti-Flag M2 (Sigma, F3165), anti-GFP JL-8 (Clontech, 8371-2), antiHA F-7 (Santa Cruz, E289), anti-actin (Sigma, A2066), antiXaf1 (IMGENEX, IMG-379), and anti-XIAP (Stressgen, AAM-50). The membranes were then washed, and incubated for 1 hour with horseradish peroxidase (HRP)-conjugated anti-mouse or anti-rabbit antibodies (Amersham Biosciences, NA931V/NA934V). Visualization of protein bands was accomplished using enhanced chemiluminescence (Amersham Biosciences) according to the manufacturer's instructions.

\section{Immunofluorescence microscopy}

Cells grown on cover slips were fixed in $4 \%$ paraformaldehyde for 10 minutes at room temperature, rinsed three times in PBS, permeabilized with cold $0.2 \%$ TritonX-100/PBS for 5 minutes, and then blocked for 2 hours (3\% BSA and 10\% FBS in PBS) at room temperature. Cells were incubated with various antibodies (anti-HA F-7 Santa Cruz E289, antiHILP/XIAP BD Transduction Laboratories 610763, at 1:200 dilution) for 2 hours at room temperature, rinsed three times in PBS, and then incubated with anti-mouse Alexa 488 or 
anti-rat Alexa 594 (Molecular Probes, A11008/A11007) 1:200 for 1 hour at room temperature. Cells were then washed, and stained with DAPI (30 $\mathrm{nM})$ for $10 \mathrm{~min}$ at room temperature prior to mounting on glass slides using anti-fade (Molecular Probes).

\section{DNA content analysis}

Saos-Xaf1 inducible cells $\left(3 \times 10^{5}\right)$ were treated as indicated with doxycycline (Fisher, $2 \mu \mathrm{g} / \mathrm{ml}$ ), TNF $\alpha$ (Endogen, $2 \mathrm{ng} / \mathrm{ml}$ ), CHX (Fisher, cycloheximide, $4 \mu \mathrm{g} / \mathrm{ml}$ ) Apo2L/ Trail (Cellsciences, $4 \mu \mathrm{g} / \mathrm{ml}$ ), Z-VAD-fmk (CalBiochem, $50 \mu \mathrm{M}$ ) and Adriamycin (Sigma, $2 \mu \mathrm{g} / \mathrm{ml}$ ). Floating and attached cells were collected, pooled washed in PBS and fixed with methanol for 2 hours at $4^{\circ} \mathrm{C}$. The cells were washed in PBS and re-suspended in PBS supplemented with propidium iodide (Fisher, $50 \mu \mathrm{g} / \mathrm{ml}$ ) and RNAse A (Sigma, $50 \mu \mathrm{g} / \mathrm{ml}$ ). DNA content was assessed by flow cytometry as previously described [20], and cells with less than 2N DNA content are scored as apoptotic. Apoptosis in transfected cells is measured in the same manner, with identification of transfected cell by co-transfection of a CD20 expression plasmid and staining as previously described [20].

\section{Cell fractionation}

Cells $\left(1 \times 10^{6}\right)$ were isolated by trypsinization. The cell pellets were washed in ice cold PBS and re-suspended in $200 \mu \mathrm{l}$ buffer A (10 mM Hepes (pH 7.9), 10 mM KCl, 0.1 mMEDTA, $0.1 \mathrm{mM}$ EGTA, $1 \mathrm{mM}$ DTT, $0.5 \mathrm{mM}$ PMSF and complete proteinase inhibitor (Calbiochem), and incubated on ice for 15 minutes. After addition of $12.5 \mu 110 \%$ NP-40, the pellets were vortexed for 15 seconds and spun at $13000 \mathrm{rpm} 1$ minute at $4{ }^{\circ} \mathrm{C}$ in a microfuge. Supernatants containing cytoplasmic protein were collected. The pellets were washed 3 times with buffer A, vortexed for 15 min after addition of $30 \mu \mathrm{l}$ buffer $\mathrm{C}$ (20 mM Hepes (pH 7.9), 420 mM NaCl, 1 mM EDTA, 1 mM EGTA, $1 \mathrm{mM}$ DTT, 1mM PMSF and proteinase inhibitors) centrifuged $13000 \mathrm{rpm}$ for $5 \mathrm{~min}$ at $4^{\circ} \mathrm{C}$. The supernatant containing nuclear proteins was collected [21]. The nuclear and cytoplasmic fractions were resolved by SDS PAGE and analyzed by western blot.

\section{Cytochrome c flow cytometery assay}

Cells $\left(2 \times 10^{5}\right)$ were collected by trypsinization, rinsed in $\mathrm{PBS}$, and incubated in $200 \mu \mathrm{l}$ digitonin solution (digitonin $50 \mu \mathrm{g} / \mathrm{ml}$ (Calbiochem), $100 \mathrm{mM} \mathrm{KCl}$ in PBS) on ice for $5 \mathrm{~min}$. Cells were fixed using $500 \mu \mathrm{l}$ of $4 \%$ paraformadehyde at room temperature for 20 minutes. Cells were washed three times in PBS, blocked for 1 hour in $500 \mu$ l blocking buffer (3\% BSA, 0.05\% Saponin (Sigma) in PBS), followed by incubation with anti-cytochrome c antibody 1:200 (Pharmingen, $6 \mathrm{H} 2 . \mathrm{B} 4)$ overnight at $4^{\circ} \mathrm{C}$. Cells were washed three times with PBS and incubated for 1 hour in $50 \mu \mathrm{l}$ of anti-Ig-FITC (DAKO, F0479) diluted 1:10 in PBS. The cells were rinsed in PBS, and re-suspended in $400 \mu$ l of PBS containing RNAse $\mathrm{A}(50 \mu \mathrm{g} / \mathrm{ml})$ and propidium iodine $(50 \mu \mathrm{g} / \mathrm{ml})$ and incubated at room temperature for 1 hour. Measurement of DNA content was assessed in FL2 and cytochrome c fluorescence was measured in FL1 using a Becton Dickenson FACScalibur.

\section{Results}

Xaf1 induces apoptosis in Saos-2 cells

We established HA-Xaf1 inducible cell lines in the human osteosarcoma tumor cell line Saos-2. Five clones that were capable of significant induction of Xaf1 in response to doxycycline treatment were analyzed in initial experiments, and compared to clones containing only empty vector. Treatment of empty vector controls demonstrated no effect of doxycycline on the growth or apoptosis of Saos 2 cells (data not shown). All Xaf1 clones behaved in a similar fashion, and the results from a typical clone are presented. Treatment of doxycycline results in accumulation of detectable levels of Xaf1 by 8 hours and as previously reported (Fig. 1A),Xaf1 is located throughout the nucleus [1], however, we also see predominant staining in sub-nuclear spots that have the appearance of nucleoli (Fig. 1B).

To determine if Xaf1 expression modulates cell cycle distribution and/or induces apoptosis in Saos2 cells, Xaf1 was induced and DNA content was measured by propidium iodide staining and flow cytometry (Fig. 1C and D). Apoptotic cells (those with less than 2N DNA content) begin accumulating at 12 hours after doxycycline treatment and increase to around $20-25 \%$ at 24 hours. In contrast, no significant effect on the cell cycle distribution is seen, with the small changes in proportion of cells in G1, S, and G2/M most likely an indirect consequence of the loss of cells by apoptosis.

\section{Xafl cooperates with TNF $\alpha$ in the induction of apoptosis}

XIAP is a broad specificity inhibitor of apoptosis, blocking cell death induced by both the death receptor and mitochondrial apoptotic pathways by direct interaction with caspases [13]. Relief of XIAP inhibition of caspase activity by Xaf1 expression would be predicted to cooperate with multiple inducers of apoptosis. This is supported by work from other investigators, indicating that Xaf1 enhances apoptosis induced by a variety of agents $[1,18]$. 
A

\section{Dox treatment (hours)}

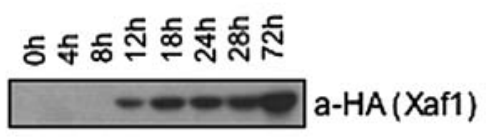

B
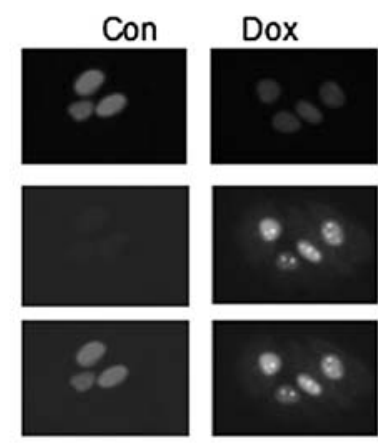

DAPI

a-HA (Xaf1)

merge
C

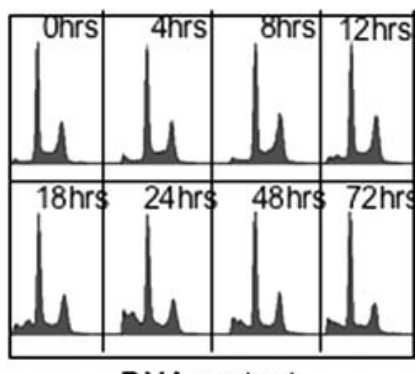

DNA content

D

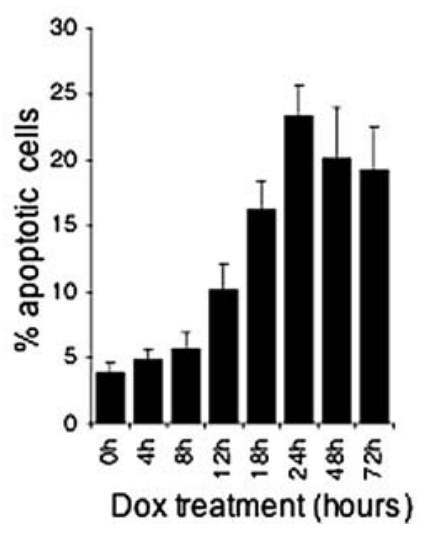

Fig. 1. Xaf1 induces apoptosis. Xaf1 tetracycline inducible cell lines were generated in Saos-2 cells. (A) Western blot showing accumulation of Xaf1 after addition of doxycycline for the times indicated. (B) Immunofluorescence of cells treated (where indicated) with doxycycline for 18 hours. Cells are stained with anti-HA (Xaf1 Green) and DAPI (Blue). (C) Flow cytometric profiles showing DNA content (x-axis) against cell number (y-axis) for Xaf1 inducible cells treated with doxycycline for the times indicated in the figure. (D) Average percentage of apoptotic cells (cells with less than 2N DNA content scored as apoptotic) after doxycycline treatment for the times indicated in the figure.

We tested if Xafl expression cooperated with the death receptor ligands TNF $\alpha$ and Apo2L/TRAIL in the induction of apoptosis in Saos- 2 cells. Low levels of TNF $\alpha$ do not induce apoptosis in Saos-2 cells (2 $\mathrm{ng} / \mathrm{ml})$ [22], however, in combination with the inhibition of protein synthesis, these cells undergo dramatic apoptosis indicating the presence of functional receptors (Fig. 2A and 2B).In the Xaf1 inducible cell lines, treatment with TNF $\alpha$ induces around $10 \%$ apoptosis in the absence of doxycycline treatment (level in parental Saos 2 and control lines is 2-3\%) and this is increased to $50 \%$ after induction of Xaf1 for 24 hours. This rate of apoptosis is sufficient to eliminate almost all of the cells in culture. The observation that Xaf1 clones have increased sensitivity to apoptosis compared to the empty vector controls in the absence of doxycycline is presumably a consequence of some 'leakiness' in the system, suggesting that low levels of Xaf1 undetectable by western blot inhibit cell survival. In contrast to TNF $\alpha$, this level of Apo2L/TRAIL does not significantly cooperate with Xaf1 in the induction of apoptosis, even though inhibition of protein synthesis was capable of rendering these cells extremely sensitive to this ligand (Fig. 2A and 2B). Increasing levels of Apo2L leads to an in- duction of apoptosis that was enhanced by Xaf1 expression, although the cooperation was not as dramatic as with $\mathrm{TNF} \alpha$ (data not shown).

Xaf1 expression rendered Saos2 cells sensitive to UV induced apoptosis. In contrast, Adriamycin was unable to cooperate with Xaf1 in the induction of apoptosis. Despite Adriamycin inducing significant apoptosis alone, expression of Xaf1 did not increase the level of apoptosis (Fig. 2A and 2B).

\section{XIAP independent function of Xafl}

To determine if the synergy between Xaf1 and TNF $\alpha$ was a consequence of an inhibition of XIAP function we first determined if these proteins were co-localized in the cell. Xaf1 is a nuclear protein and can re-localize XIAP from the cytosol to the nucleus [1]. In absence of doxycycline treatment, the sub-cellular localization of XIAP was determined to be in the cytosol, with staining typical for that seen in other cell types for this IAP. Induction of Xaf1 expression did not change the localization or staining pattern of XIAP (Fig. 3A).To confirm these results and obtain a more quantitative measure of the 
A

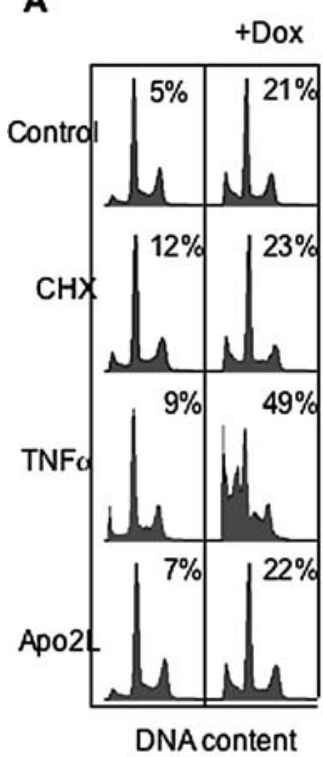

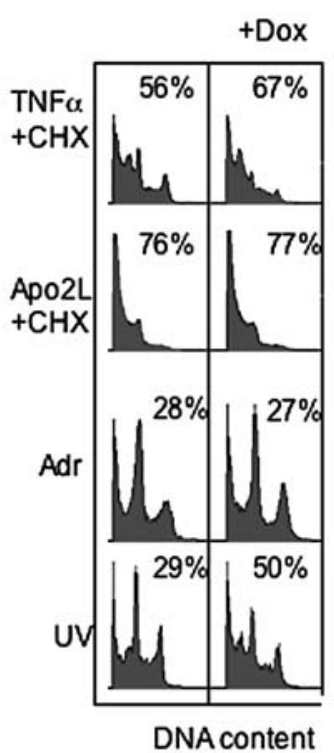

B

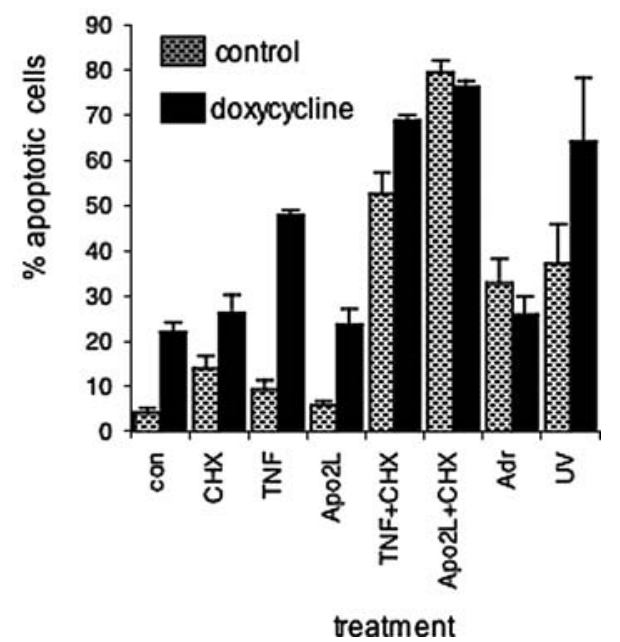

Fig. 2. Xaf1 cooperates with TNF $\alpha$ in the induction of apoptosis. (A) Flow cytometric profiles showing DNA content (x-axis) against cell number (y-axis) for Xaf1 inducible cells treated with doxycycline (DOX), cycloheximide (CHX), TNF $\alpha$, Apo2L, Adriamycin and UV as indicated as indicated in the figure. The number in each box indicates percentage of apoptotic cells. (B) Average levels of apoptosis for Xaf1 inducible cells treated with the agents indicated in the Fig., with (solid bar) and without (hatched) doxycycline treatment.

location of Xaf1 and XIAP we treated the Xaf1 inducible cells with doxycycline, $\mathrm{TNF} \alpha$ and doxycycline and $\mathrm{TNF} \alpha$ together and fractionated the cells. Total cell extract indicates that the levels of XIAP are unchanged by either Xaf1 expression or Xaf1 and TNF $\alpha$ treatment in combination (Fig. 3B). Comparison of the nuclear and cytoplasmic fractions indicated that almost all detectable XIAP is in the cytosol and all almost all Xaf1 is in the nucleus (Fig. 3C). Even under conditions where Xaf1 is dramatically enhancing $\mathrm{TNF} \alpha$ induced apoptosis, Xaf1 and XIAP are not co-localized.

One concern may be that in the generation of the lines, there may have been selection against cells in which Xaf1 and XIAP co-localized, since Xaf1 reduced colony formation relative to empty vector control plasmid. To test this, we transiently expressed HA-Xaf1 and Flag-XIAP in the parental Saos- 2 cells, and performed immuno-precipitation to determine if there was any detectable interaction in this cell type. No interaction could be detected, nor can any co-localization be seen (Fig. 3D and data not shown).

These experiments provide strong evidence that Xaf1 is inducing apoptosis independently of XIAP. However, it is possible that a small fraction of total Xaf1 is sufficient to inhibit XIAP function in the cytosol. To eliminate this possibility we utilized fibroblasts derived from XIAP deficient mice [23]. These cells were transfected with Xaf1 and control vector expression constructs and treated with $\mathrm{TNF} \alpha$ for 24 hours. Transfected cells were identified by staining pos- itive for the transfection marker CD20, and apoptosis was assessed by measuring DNA content. Xaf1 expression increased the sensitivity of these fibroblasts to TNF $\alpha$ (Fig. 3E), clearly demonstrating a XIAP independent function of Xaf1.

Xafl and Xafl+TNF $\alpha$ induced apoptosis require activation of the mitochondrial pathway

Although for convenience the death receptor and mitochondrial pathways are described separately, many cells require amplification of the apoptotic signal generated by death receptors through activation of the intrinsic pathway [24]. In some cell types this linking of the pathways is provided by the caspase 8 mediated cleavage of the $\mathrm{BH} 3$-only protein BID, this cleaved form translocates to the mitochondria triggering mitochondrial outer membrane permeabilization (MOMP) and release of cytochrome c [25, 26]. To determine if there is a requirement for activation of the mitochondrial apoptotic pathway for Xaf1 or Xaf1 and $\mathrm{TNF} \alpha$ in combination to induce apoptosis, we transiently transfected the Xaf1 inducibles with Bcl2 expression constructs. The cells were treated with doxycycline, TNF $\alpha$ or both as indicated (Fig. 4), and apoptosis was assessed in the transfected population. Baculovirus p35 was utilized as a positive control, functioning as a broad specificity caspase inhibitor [27], and inhibited apoptosis induced by both Xaf1 expression and $\mathrm{Xaf} 1$ and $\mathrm{TNF} \alpha$ in combination. Expression of Bcl2 


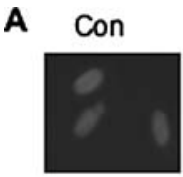
Dox
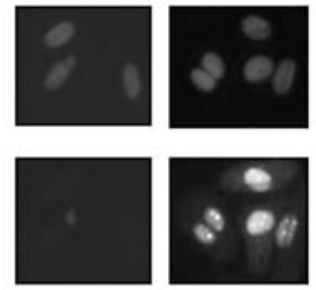

$\alpha-\mathrm{HA}($ Xaf1)
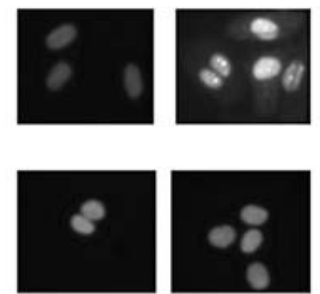

DAPI
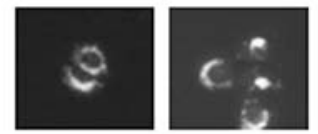

$\alpha-X] A P$
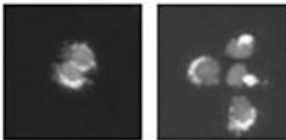

merge

B

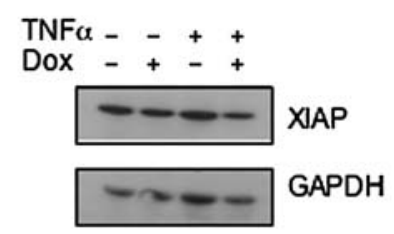

C

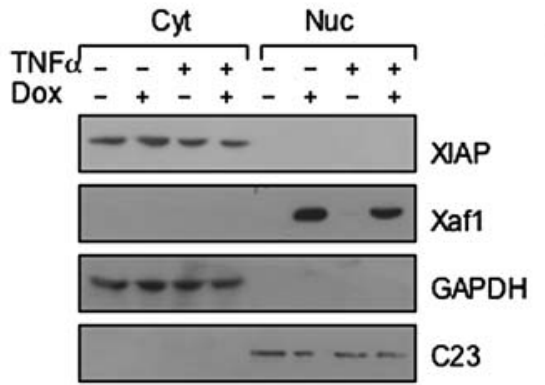

D

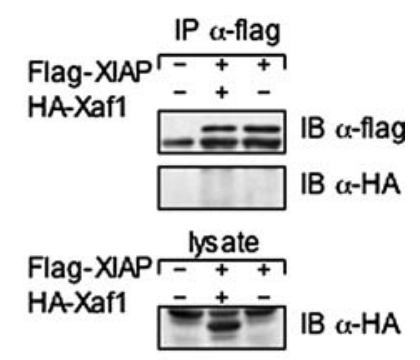

E

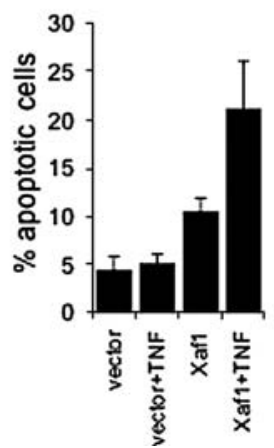

Fig. 3. XIAP independent function of Xaf 1 (A) Immunofluorescence of cells treated (as indicated) with doxycycline for 18 hours. In the top three panels, cells are stained with anti-HA (Xaf1 Green) or anti-XIAP and DAPI (Blue). In the lower three panels cells are stained with anti-XIAP (Green) or anti-XIAP and DAPI (Blue). (B) Western blot showing XIAP levels after treatment with doxycycline, TNF $\alpha$, doxycycline and TNF $\alpha$ for 24 hours. (C) Xaf1 inducible cells were treated with doxycycline, $\mathrm{TNF} \alpha$, doxycycline and $\mathrm{TNF} \alpha$ for 24 hours as indicated in the figure. Western blot showing the levels of Xaf1 and XIAP in the nuclear and cytoplasmic fractions. Successful separation of nuclear and cytoplasmic fractions is verified by blotting for GAPDH (cytoplasmic) and C23 (nuclear). (D) Extracts of Saos 2 cells transfected with plasmids encoding Flag-XIAP and HA-Xaf1 as indicated were immuno-precipitated with $\alpha$-Flag and immuno-blotted with $\alpha$-HA and $\alpha$-Flag as indicated. Lower panel is a western blot using extract corresponding to $10 \%$ of protein used in the immuno-precipitation. (E) XIAP- $/-$ mouse fibroblasts were transiently transfected with plasmids driving expression of Xaf1 or control empty vector and pCMV-CD20. The cells were treated with $\mathrm{TNF} \alpha(20 \mathrm{ng} / \mathrm{ml})$ for 24 hours, and apoptosis was measured by flow cytometry (those cells with less than 2N DNA content are scored as apoptotic). The graph expresses the average apoptotic rate for cells expressing CD20.

also inhibited all apoptosis triggered by Xaf1, and Xaf1 and $\mathrm{TNF} \alpha$ in combination demonstrating that the apoptosis induced by Xaf1 requires engagement of the mitochondrial apoptotic pathway (Fig. 4).

\section{Xaf1 triggers cytochrome c release}

$\mathrm{Bcl} 2$ inhibition of Xaf1 induced apoptosis indicates a requirement for activation of the mitochondrial pathway. Although activation of the mitochondrial pathway by death receptors is caspase dependent, activation of this pathway can occur in a caspase independent manner. For example p53 induces expression of the BH3 only proteins PUMA and Noxa that antagonize $\mathrm{Bcl} 2$ function, promoting cytochrome $\mathrm{c}$ release $[28,29]$. If Xaf 1 is functioning only to relieve IAP mediated repression of caspase activity, then we would predict that caspase activity would be necessary for the Xaf1 mediated engagement of the mitochondrial pathway, leading to the release of cytochrome $\mathrm{c}$ and other pro-apoptotic molecules. By use of the pan-caspase inhibitor z-VAD, we can determine if caspase activity is necessary for the release of cytochrome $\mathrm{c}$ from the mitochondria.

As a quantitative measure of cytochrome c release we utilized flow cytometry. Digitonin permeabilization of the cell membrane allows cytochrome $\mathrm{c}$ that is not retained in the mitochondria to be leached out of the cell [30]. By minor modification of this protocol, we could measure DNA content and cytochrome c fluorescence on the same cells, allowing us to demonstrate the effectiveness of z-VAD caspase inhibition. Since DNA fragmentation is dependent on caspase activity, $\mathrm{z}$-VAD blocks DNA fragmentation even if cytochrome $\mathrm{c}$ has been released from the mitochondria. We treated the cells with doxycycline for 18 hours and measured cytochrome c release and DNA content. As a control we treated cells with cycloheximide (CHX) and $\mathrm{TNF} \alpha$, as this results in a caspase dependent release of cytochrome c. Xaf1 expression triggered cytochrome c release, as more dramatically 


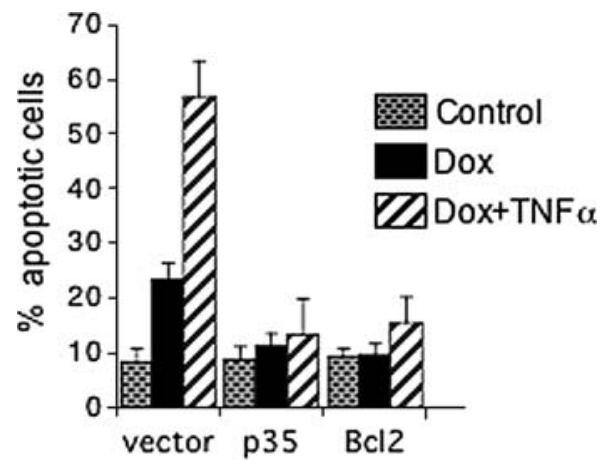

Fig. 4. Bc12 inhibits Xaf1 induced apoptosis. The Xaf1 inducible cells were transiently transfected with $\mathrm{p} 35, \mathrm{Bcl} 2$ or control empty vector expression plasmids and pCMV-CD20. The cells were treated with doxycycline $(2 \mu \mathrm{g} / \mathrm{ml}), \mathrm{TNF} \alpha(2 \mathrm{ng} / \mathrm{ml})$, or TNF $\alpha$ and doxycycline 24 hours and apoptosis was measured by flow cytometry. The graph expresses the average apoptotic rate for cells expressing CD20.

did $\mathrm{Xaf} 1+\mathrm{TNF} \alpha$ and $\mathrm{CHX}+\mathrm{TNF} \alpha$ treatment (Fig. 5A and B).Addition of z-VAD efficiently blocked cytochrome $\mathrm{c}$ release of the $\mathrm{CHX}+\mathrm{TNF} \alpha$ treated cells, in contrast the Xaf1 mediated release of cytochrome $\mathrm{c}$ was unaffected by $\mathrm{z}-\mathrm{VAD}$ treatment (Fig. 5A and B). However, z-VAD treatment resulted in complete inhibition of Xaf1 mediated DNA fragmentation, indicating successful inhibition of caspase activity by z-VAD (Fig. 5B). Although TNF $\alpha$ and CHX induced dramatic release of cytochrome $\mathrm{c}$, this was fully inhibited by z-VAD (Fig. 5C shows the results normalized, indicating almost $100 \%$ inhibition of cytochrome $\mathrm{c}$ release for $\mathrm{TNF} \alpha$ and CHX treatment).

Treatment of cells with doxycycline and TNF $\alpha$ generates a similar level of DNA fragmentation and cytochrome $\mathrm{c}$ release as TNF $\alpha+\mathrm{CHX}$. In contrast although z-VAD treatment blocked DNA fragmentation efficiently, cytochrome c release was only partially blocked. This result is what we would predict as although TNF $\alpha$ promotes cytochrome $\mathrm{c}$ release by a caspase dependent mechanism, Xaf1 mediated cytochrome c release was not blocked by z-VAD. This data suggests that Xaf1 facilitates cytochrome c release independently of caspase activation, indicating that Xaf1 has apoptotic properties in addition to the relief of XIAP-mediated (or other IAP) caspase inhibition.

\section{Discussion}

We extended previous findings indicating that Xaf1 can cooperate with a number of agents in the induction of apoptosis to Saos 2 cells. Xaf1 cooperated with both $\mathrm{TNF} \alpha$ and Apo2L/TRAIL, although more dramatically with TNF $\alpha$
A

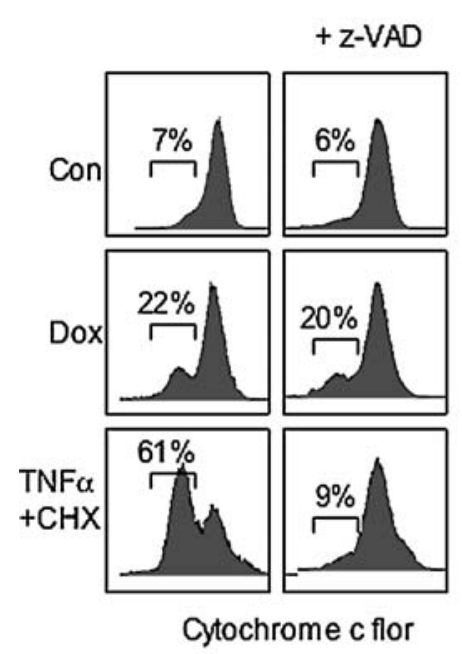

B

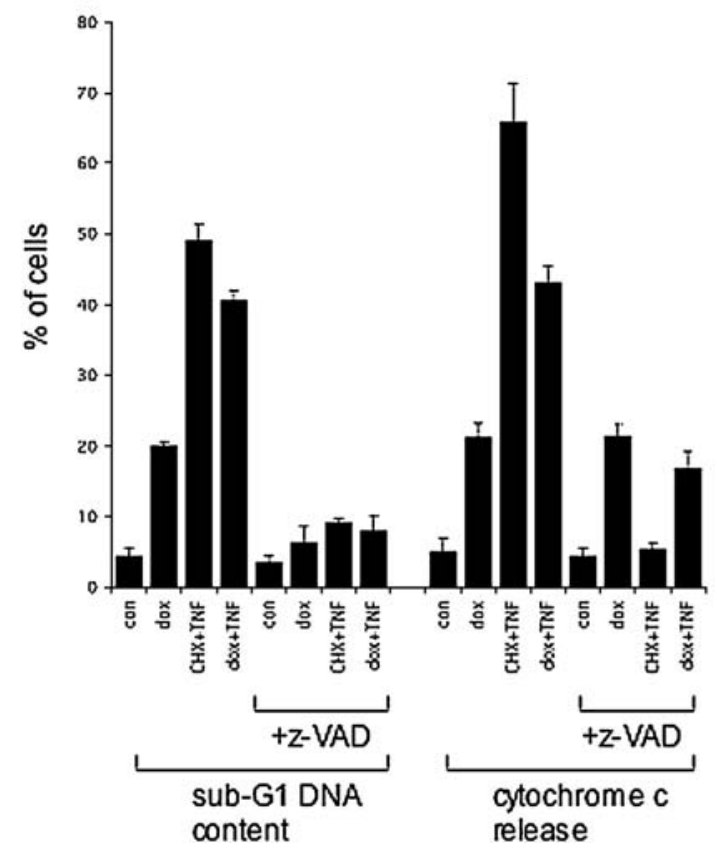

C

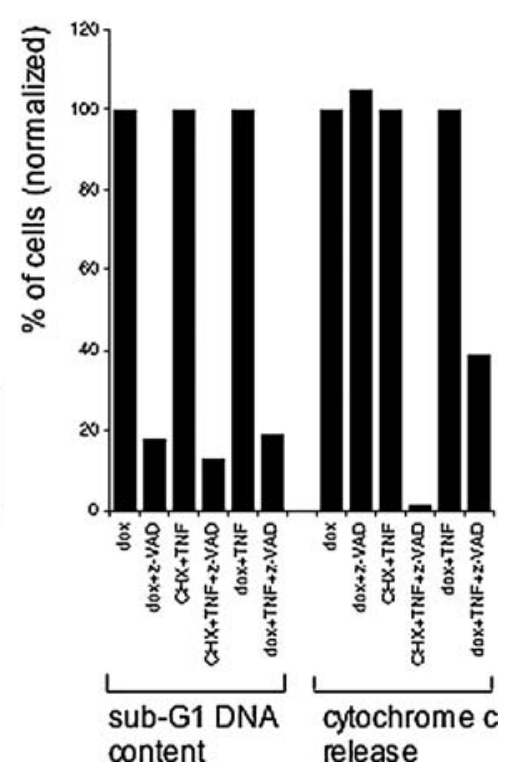

Fig. 5. Xaf1 triggers cytochrome c release by a caspase independent mechanism. The effect of z-VAD on Xaf1 induced apoptosis and cytochrome c release was measured by flow cytometry. Xaf1 inducible cells were treated as indicated with doxycycline, TNF $\alpha$, CHX, and z-VAD for 24 hours. (A) Flow cytometric profiles showing log cytochrome c fluorescence ( $\mathrm{x}$-axis) and cell number ( $\mathrm{y}$-axis). (B) Average percentage of cells with less than $2 \mathrm{~N}$ DNA content (apoptotic), and percentage of cells with released cytochrome c. (C) The results after normalization, showing relative inhibition of DNA fragmentation and cytochrome c release in the presence of $\mathrm{z}$-VAD. 
(Fig. 2 and data not shown). UV irradiation induced apoptosis was also enhanced by Xaf1 expression, however the chemotherapeutic drug Adriamycin was unable to cooperate with Xaf1, despite being capable of inducing apoptosis alone. The reason for the differences is unclear but may be related to the apoptotic pathways these agents stimulate, with Xaf1 perhaps cooperating more efficiently with death receptor signaling. Although UV can activate the mitochondrial apoptotic pathway [31-33], in some cell types UV can cross link the Fas receptor and TNFR1, independently of their ligands $[34,35]$. However, further work is required to determine if Xaf1 cooperation is limited to agents activating death receptor signaling, and why Xaf1 cooperates more efficiently with TNF $\alpha$ than Apo2L/TRAIL.

Cooperation between Xaf1 and death receptors such as TNFR could occur at a number of levels. XIAP can inhibit apoptosis upstream of MOMP in response to death receptor signaling, by preventing full processing of caspase 3 [36]. Expression of Xaf1 would be predicted to neutralize XIAP repression of caspases thus enhancing death receptor induced apoptosis. Although work from other investigators indicates that that Xaf1 can function in this manner in some cell types $[1,18]$, the data here show no evidence for a Xaf1-XIAP interaction in Saos 2 cells. Furthermore Xaf1 was able to sensitize XIAP ${ }^{-/-}$fibroblasts to TNF $\alpha$, indicating that Xaf1 can promote apoptosis independently of XIAP (Fig. 6).

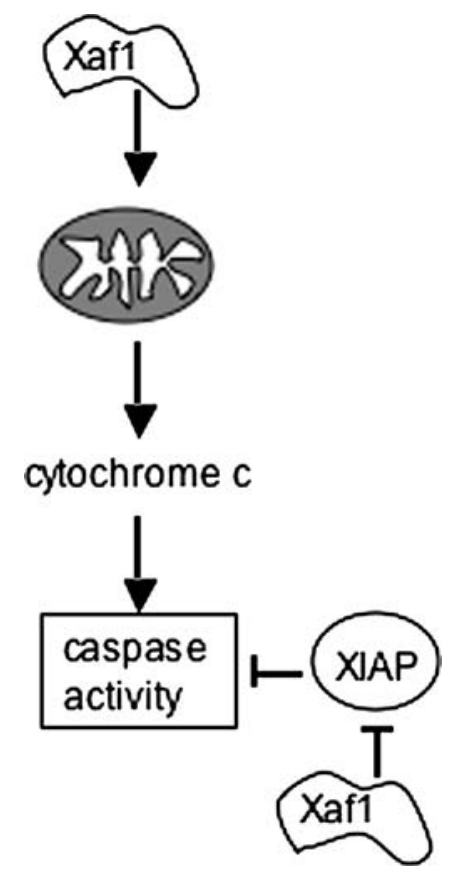

Fig. 6. Xaf1 can regulate apoptosis upstream and downstream of caspase activation. The model illustrates the points in the apoptotic cascade that Xaf1 can function. In addition to binding to XIAP reported by other investigators [1], our data demonstrate that Xaf1 can stimulate apoptosis by promoting the release of cytochrome $\mathrm{c}$ in a caspase independent manner.
Further studies are required to determine what signals control the interaction of Xaf1 and XIAP and the sub-cellular localization of these proteins, as well as the functional consequences. In addition to our data showing no co-localization, Leaman et al. have shown that interferon induced induction of Xaf1 results in Xaf1 accumulation in both nucleus and cytoplasm, however XIAP remains cytoplasmic [18]. XIAP has been reported in the nucleus in a number of papers, and interestingly can relocate from the cytosol to the nucleus in response to a number of stress signals [37-40]. Clearly in different cell types and/or under different conditions these proteins can locate to different sub-cellular compartments. An interesting possibility is that in response to certain stress conditions XIAP relocates to the nucleus to antagonize Xaf1 function, rather that Xaf1 antagonizing XIAP.

An obligatory role for the mitochondrial pathway in Xaf1 induced apoptosis is supported by the ability of Xaf1 alone to promote cytochrome c release, which cannot be inhibited by z-VAD. This data suggests that in Saos2 cells Xaf1 does not induce apoptosis by relief of IAP-mediated caspase inhibition. Further support for a role for the mitochondrial pathway in Xaf1 induced apoptosis is provided by our data demonstrating that Bcl 2 can block Xaf1 induced apoptosis. Our data implicate Xaf1 as functioning upstream of the mitochondria in a caspase independent manner. All of the data are consistent with a role in facilitating cytochrome $\mathrm{c}$ release from mitochondria to amplify apoptotic signals. This may explain, at least in part, why Xaf1 cooperates with death receptor ligands such as TNF $\alpha$, but not Adriamycin which itself can activate the mitochondrial apoptotic pathway.

How Xaf 1 promotes cytochrome c release independently of caspase activation is unclear. A direct role at the mitochondria seems unlikely since almost all detectable Xaf1 is in the nucleus. However, since Bcl2 can inhibit Xaf1 induced apoptosis it is possible that the mechanism could involve the modulation of the level, activity, or location of $\mathrm{BH}$ family protein(s). A large family of $\mathrm{Bcl} 2$ related proteins exist including proteins that inhibit or induce apoptosis including certain $\mathrm{BH} 3$ only proteins that play key roles in activating this apoptotic pathway by antagonizing Bcl2-like proteins [28, 29, 41, 42]. Since Xaf1 expression is lost in a variety of tumor types and may function as a tumor suppressor further experiments to determine how Xaf1 induces apoptosis are warranted $[2,3,5]$.

\section{Acknowledgements}

We would like to thank Krutika Haridas for technical assistance, Bob Korneluk and Peter Liston for providing the HA-Xaf1 construct and Colin Duckett, Gabriel Nunez, Alain Israel, Ed Harlow and Nancy Rice for the Flag-XIAP, p35, I-kB, CD20, and NF-kB expression constructs. The work 
was supported in part by Georgia Cancer Coalition Distinguished Scholar Award GCC00032 to A.C.P, and a University of Michigan Biological Scholars Program, Department of Defense IDEA Award PC040215 and National Institutes of Health Grant GM067827 to C.S.D.

\section{References}

1. Liston P, Fong WG, Kelly NL, Toji S, Miyazaki T, Conte D, Tamai K, Craig CG, McBurney MW, Korneluk RG: Identification of XAF1 as an antagonist of XIAP anti-caspase activity. Nature Cell Biology 3: 128-133, 2001

2. Ng KC, Campos EI, Martinka M, Li G: XAF1 expression is significantly reduced in human melanoma. J Invest Dermatol 123: 1127-1134, 2004

3. Ma TL, Ni PH, Zhong J, Tan JH, Qiao MM, Jiang SH: Low expression of XIAP-associated factor 1 in human colorectal cancers. Chin J Dig Dis 6: 10-14, 2005

4. Fong WG, Liston P, Rajcan-Separovic E, St Jean M, Craig C, Korneluk RG: Expression and genetic analysis of XIAP-associated factor 1 (XAF1) in cancer cell lines. Genomics 70: 113-122, 2000

5. Byun DS, Cho K, Ryu BK, Lee MG, Kang MJ, Kim HR, Chi SG: Hypermethylation of XIAP-associated factor 1, a putative tumor suppressor gene from the 17p13.2 locus, in human gastric adenocarcinomas. Cancer Res 63: 7068-7075, 2003

6. Brunner T, Mueller C. Apoptosis in disease: about shortage and excess. Essays Biochem 39: 119-130, 2003

7. Thornberry NA, Lazebnik Y: Caspases: enemies within. Science 281: 1312-1316, 1998

8. Slee EA, Harte MT, Kluck RM, Wolf BB, Casiano CA, Newmeyer DD, Wang HG, Reed JC, Nicholson DW, Alnemri ES, Green DR, Martin SJ: Ordering the cytochrome c-initiated caspase cascade: hierarchical activation of caspases-2, $-3,-6,-7,-8$, and -10 in a caspase-9-dependent manner. J Cell Biol 144: 281-292, 1999

9. Ashkenazi A, Dixit VM: Apoptosis control by death and decoy receptors. Curr Opin Cell Biol 11: 255-260, 1999

10. Brenner C, Kroemer G: Apoptosis. Mitochondria-the death signal integrators. Science 289: 1150-1151, 2000

11. Sharpe JC, Arnoult D, Youle RJ: Control of mitochondrial permeability by Bcl-2 family members. Biochim Biophys Acta 1644: 107-113, 2004

12. Green DR, Reed JC: Mitochondria and apoptosis. Science 281: 13091312, 1998

13. Salvesen GS, Duckett CS: IAP proteins: Blocking the road to death's door. Nature Reviews Molecular Cell Biology 3: 401-410, 2002

14. Deveraux QL, Takahashi R, Salvesen GS, Reed JC: X-linked IAP is a direct inhibitor of cell-death proteases. Nature 388: 300-304, 1997

15. Suzuki Y, Nakabayashi Y, Nakata K, Reed JC, Takahashi R: X-linked inhibitor of apoptosis protein (XIAP) inhibits caspase-3 and-7 in distinct modes. Journal of Biological Chemistry 276: 27058-27063, 2001

16. Du CY, Fang M, Li YC, Li L, Wang XD: Smac, a mitochondrial protein that promotes cytochrome c- dependent caspase activation by eliminating IAP inhibition. Cell 102: 33-42, 2000

17. Suzuki Y, Imai Y, Nakayama H, Takahashi K, Takio K, Takahashi R: A serine protease, HtrA2, is released from the mitochondria and interacts with XIAP, inducing cell death. Molecular Cell 8: 613-621, 2001

18. Leaman DW, Chawla-Sarkar M., Vyas K, Reheman M, Tamai K, Toji S, Borden EC: Identification of X-linked inhibitor of apoptosis-associated factor-1 as an interferon-stimulated gene that augments TRAIL Apo2Linduced apoptosis. J Biol Chem 277: 28504-28511, 2002

19. Bates S, Ryan KM, Phillips AC, Vousden KH: Cell cycle arrest and DNA endoreduplication following p21(Waf1/Cip1) expression. Oncogene 17: 1691-1703, 1998.
20. Phillips AC, Bates S, Ryan KM, Helin K, Vousden KH: Induction of DNA synthesis and apoptosis are separable functions of E2F-1. Genes \& Development 11: 1853-1863, 1997

21. Wadman IA, Osada H, Grutz GG, Agulnick AD, Westphal H, Forster A, Rabbitts TH: The LIM-only protein Lmo2 is a bridging molecule assembling an erythroid, DNA-binding complex which includes the TAL1, E47, GATA-1 and Ldb1/NLI proteins. Embo J 16: 3145-3157, 1997

22. Phillips AC, Ernst MK, Bates S, Rice NR, Vousden KH: E2F-1 potentiates cell death by blocking antiapoptotic signaling pathways. Molecular Cell 4: 771-781, 1999

23. Harlin H, Reffey SB, Duckett CS, Lindsten T, Thompson, CB: Characterization of XIAP-deficient mice. Molecular and Cellular Biology 21: 3604-3608, 2001

24. Green DR: Apoptotic pathways: the roads to ruin. Cell 94: 695-698, 1998

25. Luo X, Budihardjo I, Zou H, Slaughter C, Wang X: Bid, a Bcl2 interacting protein, mediates cytochrome $\mathrm{c}$ release from mitochondria in response to activation of cell surface death receptors. Cell 94: 481-490, 1998

26. Li H, Zhu H, Xu CJ, Yuan J: Cleavage of BID by caspase 8 mediates the mitochondrial damage in the Fas pathway of apoptosis. Cell 94: 491-501, 1998

27. Manji GA, Hozak RR, LaCount DJ, Friesen PD: Baculovirus inhibitor of apoptosis functions at or upstream of the apoptotic suppressor P35 to prevent programmed cell death. Journal of Virology 71: 4509-4516, 1997

28. Nakano K, Vousden KH: PUMA, a novel proapoptotic gene, is induced by p53. Molecular Cell 7: 683-694, 2001

29. Yu J, Zhang L, Hwang PM, Kinzler KW, Vogelstein B: PUMA induces the rapid apoptosis of colorectal cancer cells. Mol Cell 7: 673-682, 2001

30. Waterhouse NJ, Trapani J: A. A new quantitative assay for cytochrome c release in apoptotic cells. Cell Death Differ 10: 853-855, 2003

31. Scoltock AB, Cidlowski JA: Activation of intrinsic and extrinsic pathways in apoptotic signaling during UV-C-induced death of Jurkat cells: the role of caspase inhibition. Exp Cell Res 297: 212-223, 2004

32. Guzman E, Langowski JL, Owen-Schaub L: Mad dogs, Englishmen and apoptosis: the role of cell death in UV-induced skin cancer. Apoptosis 8: 315-325, 2003

33. Hakem R, Hakem A, Duncan GS, Henderson JT, Woo M, Soengas MS, Elia A, de la Pompa JL, Kagi D, Khoo W, Potter J, Yoshida R, Kaufman SA, Lowe SW, Penninger JM, Mak TW: Differential requirement for caspase 9 in apoptotic pathways in vivo. Cell 94: 339-352, 1998

34. Aragane Y, Kulms D, Metze D, Wilkes G, Poppelmann B, Luger TA, Schwarz T: Ultraviolet light induces apoptosis via direct activation of CD95 (Fas/APO-1) independently of its ligand CD95L. J Cell Biol 140: 171-182, 1998

35. Sheikh MS, Antinore MJ, Huang Y, Fornace AJ, Jr: Ultravioletirradiation-induced apoptosis is mediated via ligand independent activation of tumor necrosis factor receptor 1. Oncogene 17: 2555-2563, 1998

36. Wilkinson JC, Cepero E, Boise LH, Duckett CS: Upstream regulatory role for XIAP in receptor-mediated apoptosis. Mol Cell Biol 24: 70037014, 2004

37. Sauerwald TM, Betenbaugh MJ, Oyler GA: Inhibiting apoptosis in mammalian cell culture using the caspase inhibitor XIAP and deletion mutants. Biotechnol Bioeng 77: 704-716, 2002

38. Galvan V, Kurakin AV, Bredesen DE. Interaction of checkpoint kinase 1 and the X-linked inhibitor of apoptosis during mitosis. FEBS Lett 558: 57-62, 2004

39. Nowak D, Boehrer S, Brieger A, Kim SZ, Schaaf S, Hoelzer D, Mitrou PS, Weidmann E, Chow KU: Upon drug-induced apoptosis in 
lymphoma cells X-linked inhibitor of apoptosis (XIAP) translocates from the cytosol to the nucleus. Leuk Lymphoma 45: 1429-1436, 2004

40. Siegelin M, Touzani O, Toutain J, Liston P, Rami A: Induction and redistribution of XAF1, a new antagonist of XIAP in the rat brain after transient focal ischemia. Neurobiol Dis, 2005

41. Oda E, Ohki R, Murasawa H, Nemoto J, Shibue T, Yamashita T, Tokino T, Taniguchi T, Tanaka N: Noxa, a BH3-only member of the Bcl-2 family and candidate mediator of p53-induced apoptosis. Science 288 1053-1058, 2000

42. Jeffers JR, Parganas E, Lee Y, Yang C, Wang J, Brennan J, MacLean KH, Han J, Chittenden T, Ihle JN, McKinnon PJ, Cleveland JL Zambetti GP: Puma is an essential mediator of p53-dependent and -independent apoptotic pathways. Cancer Cell 4: 321-328, 2003 